\title{
Second Order Perturbation Theory for
}

\section{Generalized Active Space Self-Consistent-Field}

\section{wave functions}

Dongxia Ma, ${ }^{*, \dagger}$ Giovanni Li Manni, ${ }^{* \dagger}$ Jeppe Olsen, ${ }^{\ddagger}$ and Laura Gagliardi*,

†Max-Planck-Institut für Festkörperforschung, Heisenbergstraße 1, 70569 Stuttgart,

Germany

$\ddagger$ Department of Chemistry, Aarhus University, Langelandsgade 140, 8000 Aarhus,

Denmark

\Department of Chemistry, Supercomputing Institute, and Chemistry Theory Center, University of Minnesota, Minneapolis, MN 55455-0431, USA

E-mail: d.ma@fkf.mpg.de; g.limanni@fkf.mpg.de; gagliard@umn.edu

Supplementary Information 
Table 1: Absolute Energies (a.u.) for the chromium dimer at various geometries $[\AA]$ and levels of theory.

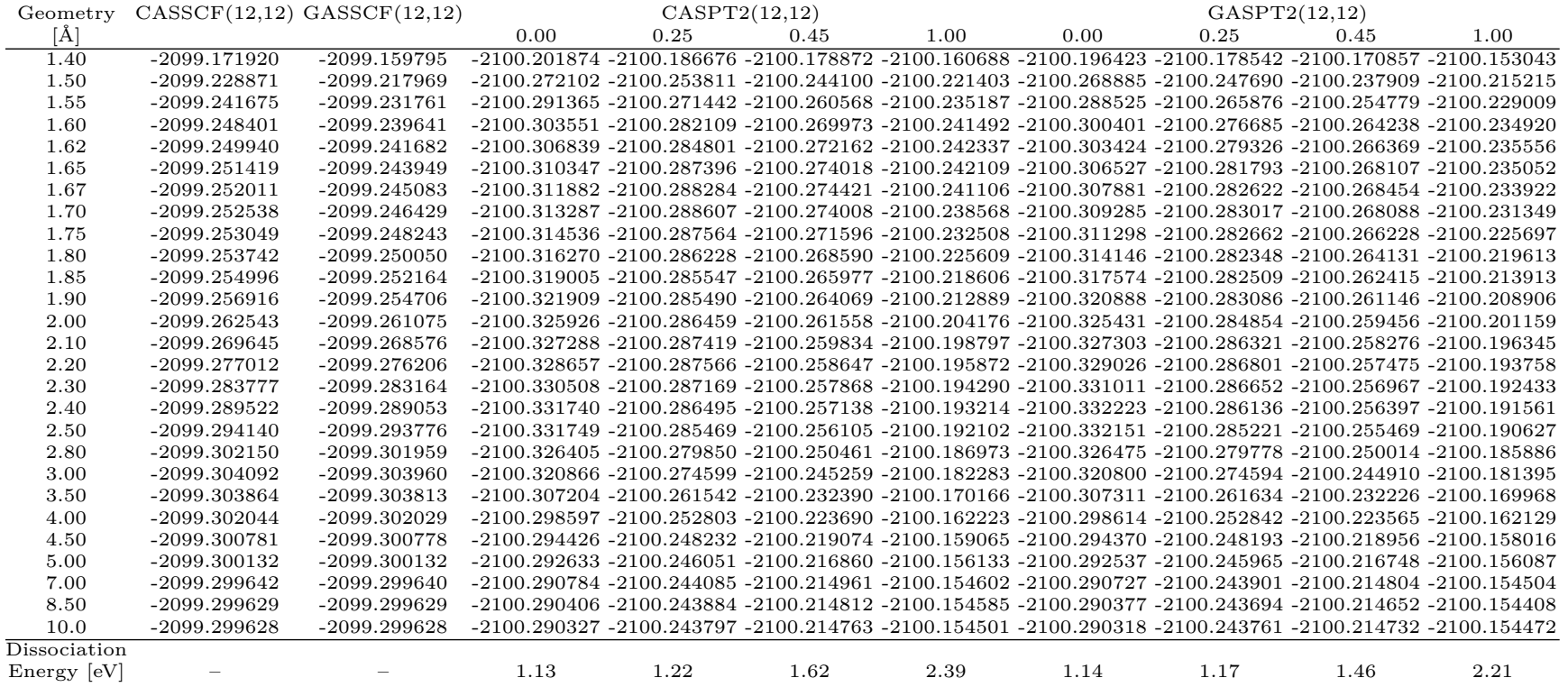

Cr dimer: ANO-RCC-V7ZP

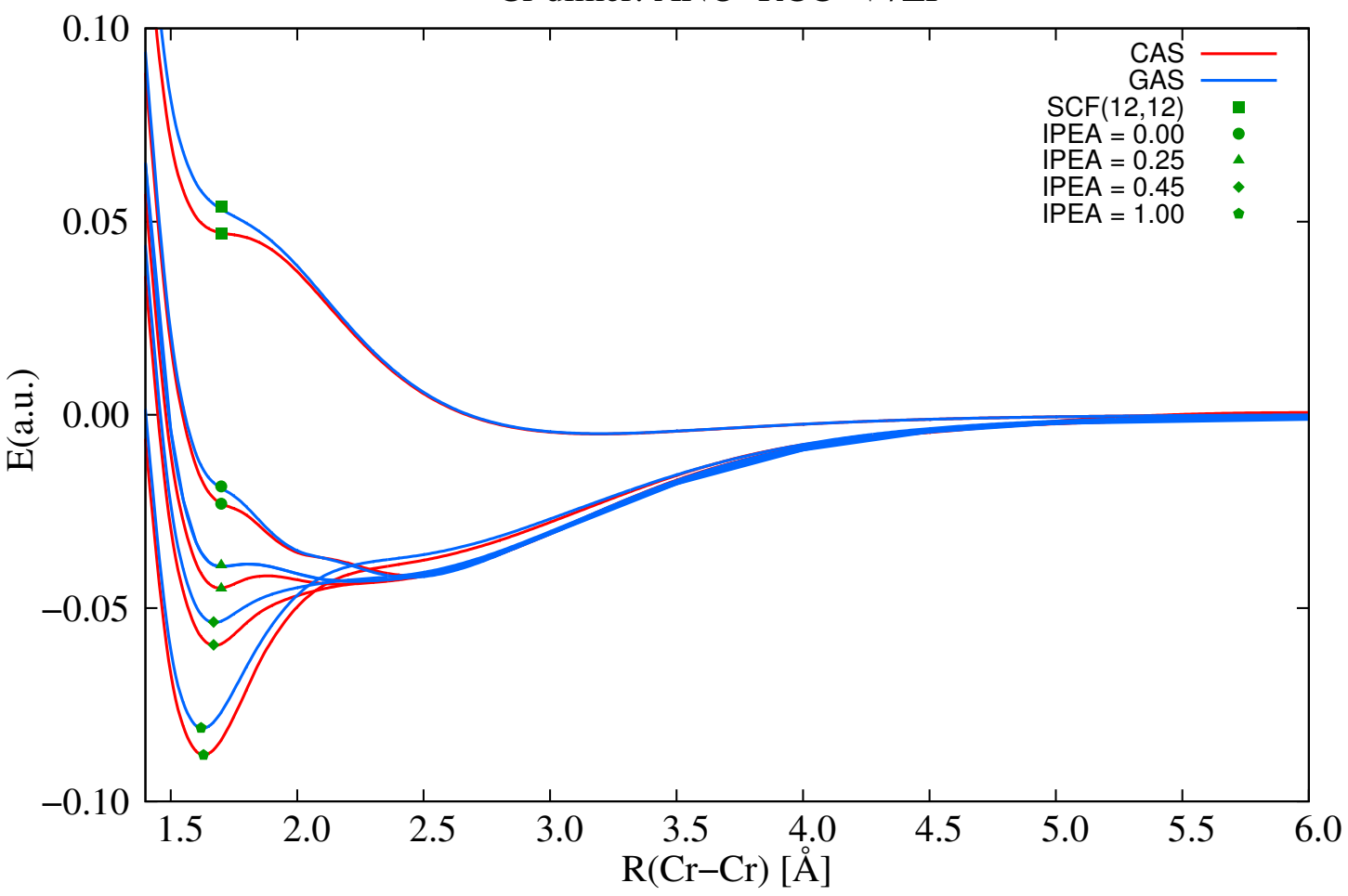

Figure 1: Potential energy curves for the $\operatorname{Cr}$ dimer for $\operatorname{CASSCF}(12,12), \operatorname{GASSCF}(12,12)$ and corresponding CASPT2 and GASPT2 with four choices of IPEA shift, 0.0, 0.25, 0.45, 1.0 a.u. Curves are shifted so that their absolute energy at $10 \AA$ is zero. 